\title{
Risk factors for pulmonary tuberculosis in community-acquired pneumonia
}

\author{
To the Editor:
}

We read with interest the study by CAVALLAZZ et al. [1] in which authors have devised a subset of risk factors that can efficiently predict pulmonary tuberculosis (TB) in patients of community-acquired pneumonia (CAP). Although they have improvised upon existing Centers for Disease Control and Prevention (CDC) list of risk factors, the study requires discussion regarding its practical value.

TB is a chronic disease with symptoms ranging from a few weeks to several months, while CAP is generally an acute process. Duration of presenting symptoms seems to be a strong factor differentiating TB from CAP, which has not been evaluated in the study.

The results of the study should be interpreted with caution as they may not be universally applicable. Only $85(1.2 \%)$ out of 6976 patients were from Asia, Africa and Australia [1], the regions that harbour an estimated $80 \%$ of incident cases of TB [2]. Mantoux positivity and recent exposure to TB were found to be significant risk factors for predicting TB in their study [1]. However, with $40 \%$ of the Indian population infected with Mycobacterium tuberculosis [3], its relevance seems questionable.

Pulmonary TB presents with a wide spectrum of clinical and radiological features that may affect the sensitivity and specificity of its risk factors. Asymptomatic presentation, normal chest radiographs [4], lower zone disease [5], Mantoux negativity (as in immunocompromised), co-infection with bacteria and fungus, associated comorbidities like COPD are important features that can falsely shift the diagnosis away from TB. Hence, absence of TB risk factors in pneumonia should not mean "exclusion of tuberculosis" but should be followed by a screening sputum microscopy for acid fast bacilli, especially in areas of high disease burden.

Further research is required, incorporating prospective cases from TB endemic countries, which may add new risk factors or change their hierarchy leading to better TB prediction.

@ERSpublications

In view of varied presentations, factors to predict pulmonary $\mathrm{TB}$ in pneumonia should be interpreted cautiously http://ow.ly/qxtWT

Deepak Aggarwal ${ }^{1}$, Prasanta Raghab Mohapatra ${ }^{2}$ and Prashant Chikkahonnaiah ${ }^{1}$

${ }^{1}$ Dept of Pulmonary Medicine, Government Medical College and Hospital, Chandigarh, and ${ }^{2}$ Dept of Pulmonary Medicine, All India Institute of Medical Sciences, Bhubaneswar, India.

Correspondence: D. Aggarwal, Dept of Pulmonary Medicine, Block-D, Level-5, Government Medical College and Hospital, Sector-32, Chandigarh, Pin-160030, India. E-mail: drdeepak@hotmail.com

Received: Aug 292013 | Accepted: Sept 122013

Conflict of interest: None declared.

\section{References}

1 Cavallazzi R, Wiemken T, Christensen D, et al. Predicting Mycobacterium tuberculosis in patients with communityacquired pneumonia. Eur Respir J 2013; 43: 178-184.

2 World Health Organization. Global Tuberculosis Report 2012, Geneva, WHO Press, 2012.

3 TB India 2012 Revised National TB Control Programme Annual Status Report. New Delhi, Central TB Division, Directorate General of Health Services, 2012.

4 Pepper T, Joseph P, Mwenya C, et al. Normal chest radiography in pulmonary tuberculosis: implications for obtaining respiratory specimen cultures. Int J Tuberc Lung Dis 2008; 12: 397-403.

5 Wang JY, Hsueh PR, Lee CH, et al. Recognising tuberculosis in the lower lung field: an age-and sex-matched controlled study. Int J Tuberc Lung Dis 2006; 10: 578-584. 